\title{
Heavy jet analysis within the JETSCAPE framework
}

\author{
Wenkai Fan* \\ Duke University \\ E-mail: wf39@duke.edu \\ Weiyao Ke \\ Duke University \\ E-mail: wk42@duke. edu
}

\section{Steffen A. Bass}

Duke University

E-mail: wk42@duke.edu

JETSCAPE is a modular, flexible, and extensible Monte Carlo event generator for simulating Heacy Ion collisions under various collision systems and collision energy. In this work, we continue the benchmark of such a framework in $\mathrm{p}+\mathrm{p}$ collisions under the heavy jet production sector. The JETSCAPE calculation is compared with PYTHIA calculation as well as experimental data and qualitative agreement is found.

13th International Workshop in High pT Physics in the RHIC and LHC Era (High-pT2019) 19-22 March 2019

Knoxville, Tennessee, USA

${ }^{*}$ Speaker. 


\section{Introduction}

The JETSCAPE (Jet Energy-loss Tomography with a Statistically and Computationally Advanced Program Envelope) is a modular, flexible, and extensible Monte Carlo event generator [1]. A heavy ion collision is a multi-scale problem, different theoretical descriptions of the evolution should be utilized to correctly describe the parton shower at different stages of the collision. Within the JETSCAPE framework, people can study single model performance as well as model-to-model comparisons in a more systematic manner. With the statistical package that will be released soon, large scale model-to-data comparison will also be easier to make.

The JETSCAPE framework was benchmarked by previous work [2,3] where results of jet and leading hadron dynamics (inclusive suppression, and the correlation between hard and soft sectors) at the LHC were presented. In this work, we will mainly focus on benchmarking the performance of JETSCAPE heavy (bottom) jet production in $\mathrm{p}+\mathrm{p}$ collision which provides a baseline for future comparisons in $\mathrm{A}+\mathrm{A}$ collisions.

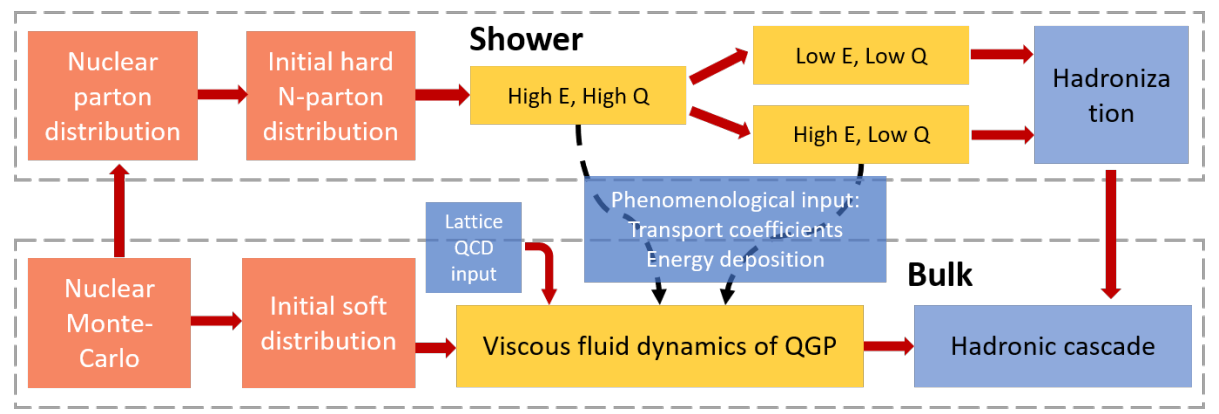

Figure 1: Simulation pipeline of the JETSCAPE framework. Each module can be modified individually.

\section{Difference between JETSCAPE and Pythia in $p+p$ collisions}

When doing $\mathrm{p}+\mathrm{p}$ collisions, JETSCAPE initializes the hard partons by calling PYTHIA [5]. The hard partons first undergo initial state radiation (ISR) and multi-parton interaction (MPI). But instead of having final state radiation (FSR) as in PYTHIA, JETSCAPE then propagates the partons using MATTER [4] which produces virtuality-ordered shower with large virtuality $\left(Q^{2} \geq \sqrt{\hat{q} E}\right.$ in medium and $Q^{2} \geq 1 \mathrm{GeV}^{2}$ in vacuum). This is the main difference between JETSCAPE and PYTHIA when it comes to simulate $\mathrm{p}+\mathrm{p}$ collisions.

\section{Results}

Figure. 2 shows the bottom jet cross-section in $\mathrm{p}+\mathrm{p}$ collisons at $2.76 \mathrm{TeV}$ from JETSCAPE, PYTHIA, and the CMS experiment. Though JETSCAPE can reproduce the inclusive jet production in $\mathrm{p}+\mathrm{p}$ collisions well [3], it seems to differ from both PYTHIA and experimental results at high $p_{T}$. This difference may due to the fact that current MATTER implementation treats all quarks as massless particles. We will redo the calculation to see if the difference is reduced when the next version of JETSCAPE, which includes the mass effect in MATTER, comes out. We can also look 

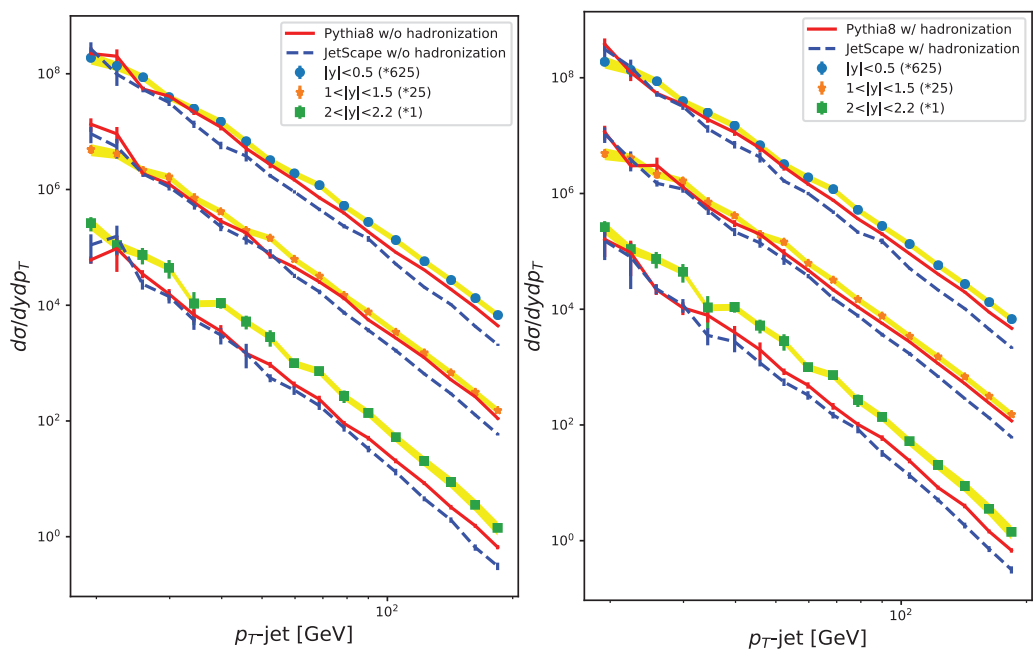

Figure 2: Bottom jet cross-section between models and experiments without and with (left / right) hadronization, for different jet rapidity (rows). The jets are constructed using an anti-kT algorithm with $R=0.4$. Data taken from Ref. [6]

at the ratio of model calculations to experimental data. Again the same trend shows: JETSCAPE produces fewer bottom quark jets at high $p_{T}$.

If we take the ratio of the cross section of bottom jets to inclusive jets, the JETSCAPE calculation is lower than experimental data at high $p_{T}$ at small rapidity, but seems to perform better than PYTHIA at high rapidity.

\section{Conclusion}

In this work we looked at a unified approach to the modeling of jet quenching called JETSCAPE. Specifically a virtuality-ordered shower for high virtuality partons (MATTER) is used to describe the evolution of high virtuality partons in $\mathrm{p}+\mathrm{p}$ collisions. The performance of such a method on charged hadrons and inclusive jets has been shown to be quite good[3]. However due to the missing physics in the current implementation, JETSCAPE predicts a smaller cross-section of bottom quark jets at high $p_{T}$.

\section{References}

[1] Kauder, Kolja, and Jetscape Collaboration. "Jetscape v1. 0 quickstart guide." Nuclear Physics A 982 (2019): 615-618.

[2] Cao, Shanshan, et al. "Multistage Monte Carlo simulation of jet modification in a static medium." Physical Review C 96.2 (2017): 024909.

[3] Park, C., et al. "Multi-stage jet evolution through QGP using the JETSCAPE framework: inclusive jets, correlations and leading hadrons." arXiv preprint arXiv:1902.05934 (2019).

[4] Majumder, Abhijit. "Incorporating space-time within medium-modified jet-event generators." Physical Review C 88.1 (2013): 014909. 

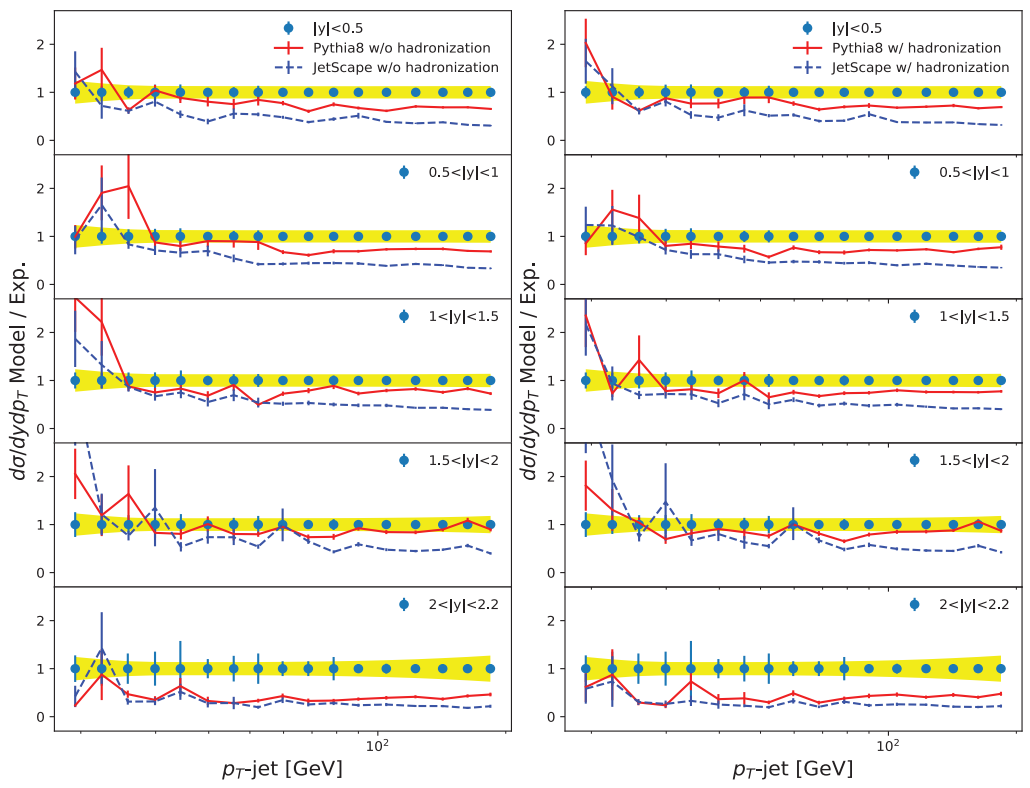

Figure 3: Ratio of bottom jet cross-section between models and experiments without and with (left / right) hadronization, for different jet rapidity (rows). The jets are constructed using an anti-kT algorithm with $R=0.4$. Data taken from Ref. [6]
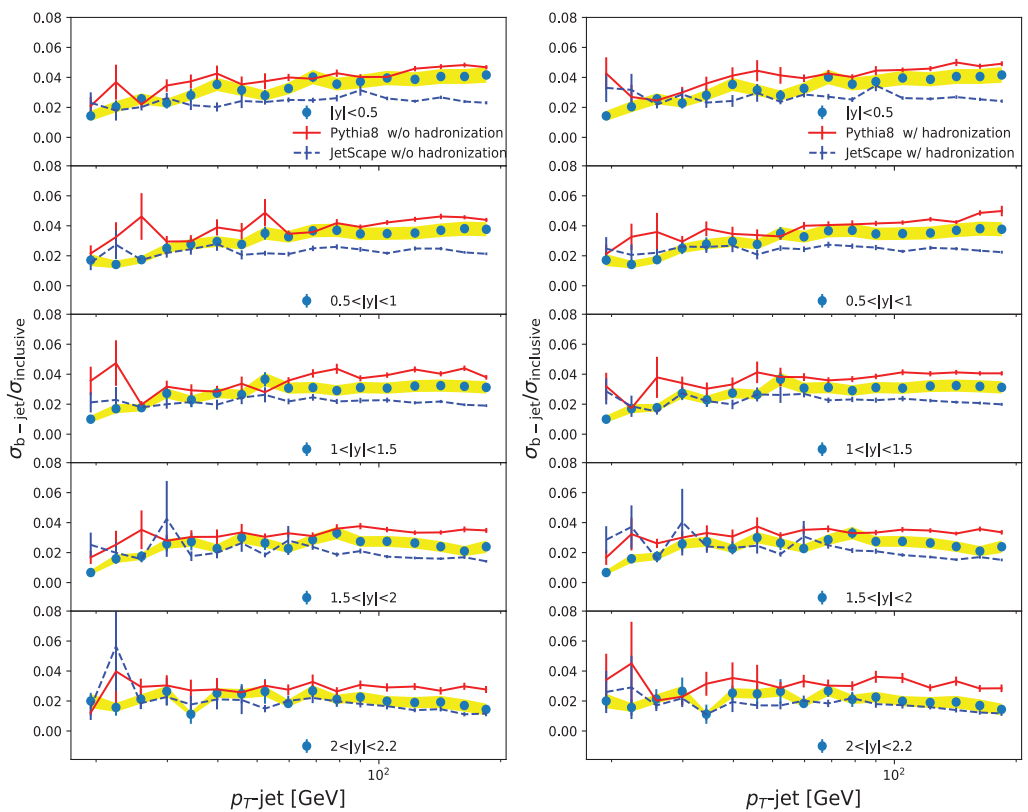

Figure 4: Ratio of bottom jet cross-section to inclusive jet cross section between models and experiments without and with (left / right) hadronization, for different jet rapidity (rows). The jets are constructed using an anti-kT algorithm with $R=0.4$. Data taken from Ref. [6] 
[5] Sjostrand, Torbjorn, Stephen Mrenna, and Peter Skands. "A brief introduction to PYTHIA 8.1." Computer Physics Communications 178.11 (2008): 852-867.

[6] CMS collaboration. "Inclusive b-jet production in pp collisions at sqrt $(\mathrm{s})=7 \mathrm{TeV}$." arXiv preprint arXiv:1202.4617 (2012). 